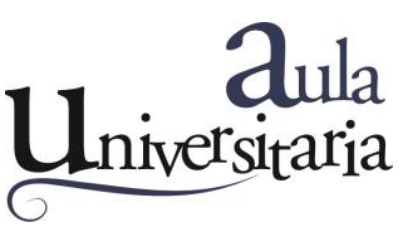

\title{
Experiencia pedagógica de promoción en alimentación saludable para estudiantes universitarios
}

\author{
CELEGHIN, Adelina G.; ${ }^{1}$ LENCINA, Florencia A.; \\ IRIBARREN, Gissel N.; ${ }^{3}$ FORTINO, María A. ${ }^{4}$
}

\begin{abstract}
Filiaciones institucionales
${ }^{1}$ Docente de Fisicoquímica y Tecnología de Alimentos. Facultad de Bioquímica y Ciencias Biológicas. Universidad Nacional del Litoral.

${ }^{2}$ Estudiante de Licenciatura en Nutrición. Formación Extracurricular en Docencia de la Facultad de Bioquímica y Ciencias Biológicas. Universidad Nacional del Litoral.

${ }^{3}$ Graduada de Facultad de Bioquímica y Ciencias Biológicas. Servicio de Actualización y Perfeccionamiento para Graduados. Universidad Nacional del Litoral.

${ }^{4}$ Docente de Química Biológica. Investigadora del Laboratorio de Estudio de Enfermedades Metabólicas relacionadas con la Nutrición. Facultad de Bioquímica y Ciencias Biológicas. Universidad Nacional del Litoral.
\end{abstract}

\section{Correspondencia}

Paraje El Pozo, s/n, 1er. Piso. Santa Fe de la Vera Cruz, Santa Fe, Argentina. Tel. 54-342-4575210 celeghin@fbcb.unl.edu.ar

\section{Resumen}

El ámbito académico cumple un rol relevante en la creación de entornos saludables para la prevención de enfermedades; es así que el aula universitaria se convierte en un espacio generador de oportunidades. En el presente artículo se discuten variables contempladas en la planificación y ejecución de una estrategia pedagógica orientada a la nutrición, en el formato de un curso dirigido a alumnos de Bioquímica y Licenciatura en Biotecnología de la Facultad de Bioquímica y Ciencias Biológicas de la Universidad Nacional del Litoral. El objetivo fue brindar educación alimentaria nutricional para promover cambios favorables en los hábitos y elecciones alimentarias de estos estudiantes. Se trabajó utilizando como eje temático a las Guías Alimentarias para la Población Argentina 2016, promoviendo la participación activa en los procesos de aprendizaje. Se 
enfatizó la aplicación de las recomendaciones de dichas guías contextualizadas al ámbito universitario, priorizando la realización de actividades prácticas y su articulación con conocimientos previos de otras asignaturas.

Los resultados indicaron que la implementación del curso incidió favorablemente en la construcción de conocimientos y habilidades referidos a alimentación y nutrición de los participantes. Puede concluirse que resultaría beneficioso trasladar estas experiencias a alumnos de otras carreras interesados en la problemática.

\section{Palabras clave}

educación alimentaria nutricional | estrategia pedagógica | curso teórico-práctico | alumnos universitarios

\section{Summary}

The academic field plays a relevant role in the creation of healthy environments and disease prevention; in this sense the university classroom becomes a space that generates opportunities. This article shows variables contemplated in the planning and execution of a pedagogical strategy oriented to nutrition, in the format of course, aimed at students of Biochemistry and Bachelor of Biotechnology of the Facultad de Bioquímica y Ciencias Biológicas, Universidad Nacional del Litoral (Santa Fe, Argentina). The subject was to provide nutritional food education to promote changes in eating habits and food choices of these students. Food Guidelines for Population of Argentina 2016 were used as thematic axis of work, promoting the active participation in learning processes. Application of recommendations from these guides was contextualized to the university field, prioritizing the realization of practical activities and their articulation with previous knowledge of these students.

The results indicated that implementation of the course had a favorable impact on the construction of knowledge and skills related to food and nutrition of the participants. It is possible to conclude it would be beneficial to apply these experiences in students from other careers who are interested in the problem.

\section{Keywords}

nutritional food education / pedagogical strategy / theoretical-practical / course / university students

\section{Introducción}

En los últimos años, uno de los principales problemas de salud que alarma a la población mundial es el gran aumento en la obesidad, que ha alcanzado proporciones epidémicas en las sociedades occidentales (OMS, 2017). Esta patología es el resultado de la combinación de factores genéticos junto a estilos de vida que promueven la alimentación inadecuada y la disminución de la actividad física regular. El sobrepeso y la obesidad no suelen ser una enfermedad solitaria, se asocian habitualmente a otras patologías denominadas en su conjunto como enfermedades crónicas no trasmisibles (ECNT). Entre las 
principales se pueden citar: diabetes tipo 2, hipertensión arterial, enfermedades coronarias, dislipemias, síndrome metabólico y ciertos tipos de cánceres; las cuales acortan la esperanza y la calidad de vida (De Girolami y González Infantino, 2008). En Argentina, 6 de cada 10 adultos presentan exceso de peso (sobrepeso y obesidad) según datos del Ministerio de Salud y Desarrollo Social (MSDS) en la última Encuesta Nacional de Factores de Riesgo (4ํENR, 2019), lo que evidencia un aumento estadísticamente significativo respecto de la anterior (2013) y sostenido desde la primera (2005). En consecuencia, las intervenciones que logren promover un cambio conductual en la alimentación y la actividad física, cobran cada vez mayor importancia, comprobándose además que adquirir los buenos hábitos a temprana edad resulta esencial en la prevención (OMS, 2018).

Teniendo en cuenta que el escenario universitario no es ajeno a esta situación, resulta de relevancia la intervención mediante estrategias pedagógicas que aborden aspectos nutricionales. Según Holli y Beto (2018) desde el punto de vista educativo las recomendaciones nutricionales resultan esenciales para promover un estilo de vida saludable y prevenir la enfermedad, siendo necesario adaptarlas al grupo poblacional al cual van dirigidas. Si bien la motivación es un potente motor de cambio de hábitos, la contextualización es un punto importante que incide en la aplicación de lo aprendido y su sostenimiento en el tiempo; de modo que ambos factores resultan clave en el diseño de acciones que potencien el éxito del proceso educativo en salud.

En trabajos diagnósticos previos de esta línea de investigación se ha determinado que estudiantes de Bioquímica (B) y Licenciatura en Biotecnología (LB) de la Facultad de Bioquímica y Ciencias Biológicas - Universidad Nacional del Litoral (FBCB-UNL), poseen hábitos alimentarios que podrían ser mejorados, así como también un menor conocimiento en alimentación saludable comparado con sus pares de Licenciatura en Nutrición (Berta et al., 2017; Vargas et al., 2017; Noseda et al., 2018). Atendiendo a ello se diseñó una estrategia, en formato de curso teórico-práctico, denominado «Alimentarnos Sanamente» destinado a alumnos de estas dos carreras. El objetivo general del mismo se orientó a la educación alimentaria nutricional basada en la práctica para lograr autonomía en la selección de alimentos y en la preparación de menús saludables.

La formación de recursos humanos capaces de replicar las experiencias en un futuro y en otros ámbitos es muy importante para la educación en salud. Paralelamente, se reconoce que la educación por pares es una herramienta valiosa (Cardozo-Ortiz, 2011). La FBCB ofrece oportunidades institucionales que fomentan la participación en las áreas de Docencia, Investigación y Extensión, entre las que se cuentan la Formación Extracurricular para Alumnos y el Servicio de Actualización y Perfeccionamiento para Graduados. Estas posibilidades se tomaron como parte de la estrategia educativa, incorporando al dictado del curso a auxiliares (alumno/graduado) de la carrera de Licenciatura en Nutrición bajo estas normativas. 
Lo que se pretende en este artículo es comunicar una experiencia pedagógica vivenciada en el contexto académico relacionada con el mantenimiento de la salud y la prevención de enfermedades de creciente impacto social; discutiendo la importancia de los contenidos abordados y generando una reflexión crítica sobre la propuesta ofrecida y las estrategias implementadas.

\section{Metodología}

\section{Planificación de la actividad educativa}

En el marco de un proyecto de educación en alimentación saludable surgieron diferentes propuestas entre las que se contó un curso específico en la temática (Fortino et al., 2019). La premisa en la planificación de las estrategias educativas fue la contextualización al ámbito de la FBCB-UNL. El desafío en la implementación del curso no solo fue captar la atención de los estudiantes para lograr la adquisición de habilidades (en este caso con la finalidad de promover la salud), sino también cómo conseguir ese activo proceso mental de construcción de saberes y elaboración de juicio crítico sobre lo aprendido, indispensable para el sostenimiento en el tiempo que requieren los hábitos saludables.

Tomando en consideración que el curso se dirigía a un grupo de estudiantes de ciencias, se abogó por contenidos y prácticas donde la incorporación de los nuevos conocimientos estuviese articulada con otros de asignaturas propias de sus carreras (química, física, termodinámica, biología, entre otras). Como guía de los procesos de enseñanza y aprendizaje se tuvo en cuenta el modelo del triángulo pedagógico, con especial interés en la investigación de la relación que se crea dentro del aula universitaria, y entendiéndola como una compleja red de interrelaciones entre estudiante-docente-conocimiento, con todas las ventajas y desventajas que el modelo implica (Ibáñez Bernal, 2007).

Respecto del espacio áulico para el desarrollo educativo se eligió el laboratorio, entendiendo al aprendizaje teórico-práctico como una unidad indisociable para los objetivos propuestos (Flores et al., 2009). En este diseño, donde el objetivo principal es que los alumnos sean partícipes activos de su formación, el laboratorio como espacio áulico permite realizar las prácticas y, en forma alternada o simultánea, la utilización de los recursos didácticos para el desarrollo teórico (power point, videos, entre otros). Además, la ubicación del grupo alrededor de la mesada de trabajo favorece el contacto visual y el permanente intercambio de los participantes entre sí y con el docente.

El número reducido de alumnos (máximo de 10) acompañados por un docente y un auxiliar (alumno/graduado), se pensó en función del espacio disponible y como facilitador en la generación de un ambiente motivador, de colaboración e intercambio descontracturado - aunque respetuoso- como parte de la estrategia. En este contexto, la educación entre pares propicia 
actitudes, comportamientos, intercambios y fines compartidos en situación de igualdad, tendiente a homogeneizar el poder de la relación y proporcionando oportunidades singulares para abordar conflictos o dificultades personales (Fernández García, 2003).

Con respecto a la evaluación, en las últimas décadas el concepto ha sufrido una profunda transformación y en la actualidad se valora el aprendizaje del alumno en el proceso y en el producto, reconociéndose que las prácticas evaluativas ya no se corresponden con un acto final sino como una parte del proceso de la enseñanza y del aprendizaje (Bordas y Cabrera, 2001). La evaluación por lo tanto debe ser consistente con la metodología de desarrollo del proceso y una parte del mismo; de este modo se propuso como una última clase práctica integradora que desafíe los aprendizajes logrados por los participantes. La misma se concibió como una actividad de carácter práctico (elaboración de un menú), donde cada uno de los estudiantes aportara los conocimientos adquiridos para arribar a una elaboración grupal conjunta.

\section{El curso «Alimentarnos Sanamente»}

La actividad educativa se formalizó como Curso de Extensión de la FBCBUNL dirigido a estudiantes de Bioquímica y Licenciatura en Biotecnología, con una carga horaria total de 24 horas distribuidas en 8 encuentros semanales de actividades teóricas y prácticas. Al presente se ha dictado en tres oportunidades: $1^{\circ}$ y $2^{\circ}$ semestre de 2018 (con la participación de una graduada) y $1^{\circ}$ semestre de 2019 (con la participación de una alumna avanzada).

Inicialmente se realizó una encuesta diagnóstica de conocimientos sobre nutrición y hábitos alimenticios, así como también del conocimiento y aplicación de las Guías Alimentarias para la Población Argentina (GAPA) por parte de los participantes. El instrumento utilizado fue el mismo que se empleó en la investigación diagnóstica del proyecto marco (Noseda et al., 2019).

La dinámica de los encuentros se desarrolló con un marco teórico inicial de los nuevos conocimientos (con eje en las recomendaciones de las GAPA), para luego realizar las actividades prácticas programadas. En todo momento se hizo hincapié en la identificación de los procesos biológicos y fisicoquímicos que ocurren durante el procesamiento de los alimentos y las buenas prácticas alimentarias, relacionándolos con aprendizajes preexistentes. Las actividades se dirigieron a:

- Desarrollar autonomía en la selección de alimentos e interpretación de los rotulados nutricionales.

- Desarrollar habilidades en el manejo de alimentos mediante la aplicación de buenas prácticas de manufactura. 
- Seleccionar y elaborar menús saludables, económicos, fáciles de preparar y adecuados para transportar y consumir en el ámbito universitario.

Atendiendo al uso de las tecnologías de información y comunicación actuales, el curso contó con el apoyo de un espacio educativo institucional creado a tal efecto en el Entorno Virtual UNL; alternativamente, se utilizó como recurso de intercambio informal a las redes sociales (Whatsapp), se creó un grupo en cada oportunidad de dictado.

En cada uno de los años en que se dictó el curso el auxiliar (graduado/alumno) contribuyó generando un aporte educativo de elaboración propia a la propuesta original.

\section{Resultados y discusión}

El registro de resultados corresponde a 21 alumnos, 9 de Licenciatura en Biotecnología y 12 de Bioquímica, con edades comprendidas entre 19 y 27 años. A pesar de existir diferencias de conocimientos básicos, relacionados al grado de avance en sus carreras (ya que participaron alumnos de 1ero. a 5to. año), esto no fue motivo de inconvenientes en la interpretación de los nuevos conocimientos ni en la conformación y convivencia de los grupos.

En el primer encuentro se conversó sobre las motivaciones personales para realizar el curso y se realizó la evaluación de conocimiento e interés mediante encuesta (valoración diagnóstica). Estos instrumentos permitieron fijar un punto de partida y adecuar los contenidos a las necesidades particulares del grupo conformado, entendiendo que los esfuerzos debían focalizarse en las falencias encontradas. En términos generales, pudo apreciarse que los participantes consideraban su alimentación como medianamente saludable y coincidían en un manifiesto interés por mejorarla. Un aspecto importante a tener en cuenta fue que, en algunos casos, en la decisión de tomar el curso influyó la referencia positiva de otros estudiantes que lo habían realizado previamente.

Con relación al conocimiento previo se reveló que: 17 alumnos (81\%) desconocían la existencia de las Guías Alimentarias para la Población Argentina (GAPA); la pirámide alimentaria fue la gráfica de alimentación saludable más reconocida (90\%), mientras que el plato contó con un reconocimiento menor $(71,4 \%)$. Estos resultados se corresponden con los previos encontrados en la población estudiantil de estas carreras (Noseda,2019), y que dieron origen a la implementación del curso.

A propuesta de la auxiliar en 2019, a fin de ampliar la valoración diagnóstica se sumó un registro de ingesta de 24 horas y un cuestionario de frecuencia alimentaria, cuya finalidad estuvo orientada hacia la reflexión de los alumnos sobre su propia alimentación. El diagnóstico nutricional evidenció que los hábitos alimentarios de los/as participantes se correspondían, en términos generales, 
con los lineamientos de una alimentación completa. En lo educativo se pudo observar cierta dificultad para el cálculo de las porciones consumidas. Esto es común y se cuenta entre las dificultades que conllevan estos tipos de encuestas, junto a otros tales como la omisión de alimentos o incluso la influencia ejercida por estos registros en el consumo del individuo (De Girolami y González Infantino, 2008). La actividad junto al docente permitió salvar las dudas y adquirir los conocimientos necesarios para valorar su propia ingesta de un modo asertivo, cumpliendo con el objetivo de estimular el pensamiento crítico para tomar decisiones adecuadas en cuanto a su alimentación.

Luego de una breve introducción teórica, la mayor parte de la clase se destinó a las actividades prácticas que consistieron en la elaboración de recetas nutritivas. Un punto a destacar fue el interés por parte de los estudiantes hacia los contenidos teóricos desarrollados y la motivación para la llevar a cabo la parte práctica, manifestando su conformidad con respecto a la selección de menús propuestos como herramientas para el aprendizaje.

Teniendo en cuenta los objetivos no se trató de un simple curso de cocina, sino que desde la ciencia se trabajó en la aplicación de técnicas de manejo e higiene de los alimentos, el manejo de equipos, la exploración de los procedimientos y el análisis de las técnicas. Un logro importante resultaron las ideas innovadoras que surgieron a partir de estas prácticas, combinando así los conocimientos adquiridos durante las clases con las habilidades individuales de cada estudiante. La capacidad para aplicar lo aprendido en nuevas situaciones da cuenta de un aprendizaje significativo. Aún más Díaz Barriga y Hernández Rojas proponen que «la función del docente es engarzar los procesos de construcción del alumno con el saber colectivo culturalmente organizado» (2010:32). Prueba de ello en los estudiantes del curso fue el relato y el registro (en ocasiones compartido mediante redes sociales), donde se plasmaron versiones saludables de snacks y comidas rápidas en las reuniones con sus pares; bizcochuelos, tortas y tartas frutales para acompañar sus horas de estudio y la mención de los pasteles y tartas de vegetales como principal opción de elaboración en sus viandas diarias. Algunos de estos registros se muestran en la figura 1. La incorporación de frutas y verduras reviste crucial importancia ya que, a pesar de reconocerse su valor nutricional, la 4a ${ }^{-}$Encuesta Nacional de Factores de Riesgo (ENFR) demostró su bajo consumo en la dieta de los argentinos. 
Universitaria
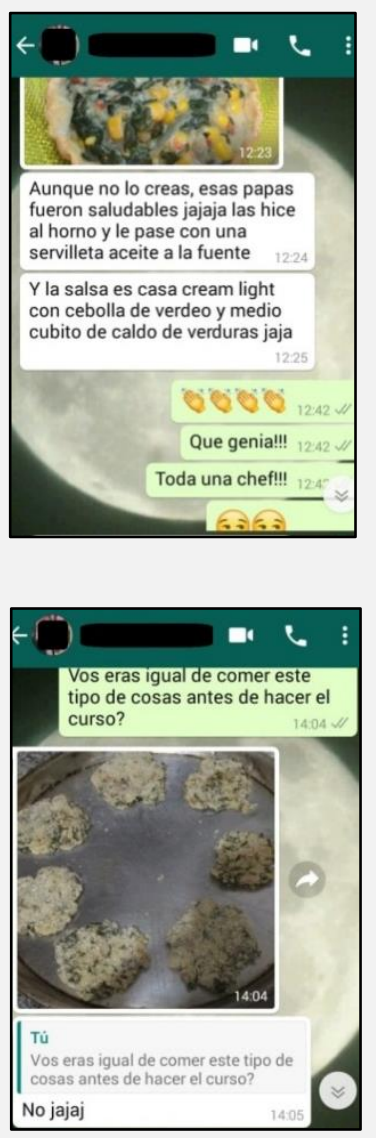
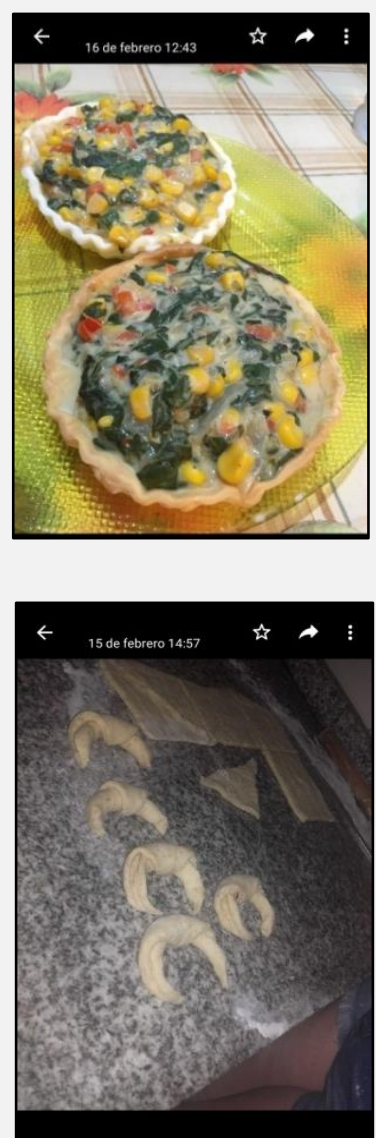
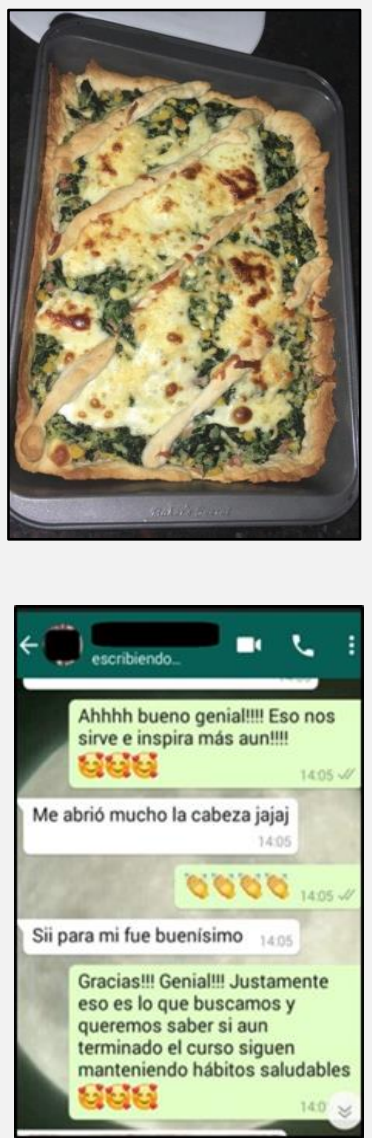

Figura 1. Capturas de pantalla con opiniones y fotografías de preparaciones compartidas por los alumnos

El uso de medidas caseras, la interpretación de los rotulados de alimentos y la activación de semillas se contaron entre las temáticas que despertaron alto interés, tanto por su desconocimiento previo, como por la marcada importancia $y$, particularmente en el caso de semillas, el auge y la gran repercusión que ha alcanzado en la actualidad. Para la clase de rotulados nutricionales, se solicitó a los participantes que buscaran rótulos en alimentos de su consumo habitual, que les causaran inquietud (duda, confusión, curiosidad, etc.) para luego analizarlos en conjunto durante la clase. Esta dinámica de trabajo arrojó resultados positivos en cuanto a la participación activa en la construcción de conocimientos, promoviendo el interés por la lectura de los mismos. En cada receta trabajada se implementó la elaboración de su rotulado nutricional bajo el mismo formato presentado por los alimentos del mercado y establecido por la Administración Nacional de Medicamentos, Alimentos y Tecnología (ANMAT). Dentro del rótulo, para dar cuenta del valor nutricional de cada receta, se utilizó el software ofrecido por el Ministerio de Salud denominado SARA (Sistema de Análisis y Registro de Alimentos). Los resultados positivos se reflejaron en el desarrollo de capacidades críticas por parte de los estudiantes, quienes expresaron su cambio de actitud durante la realización de las compras en base a los rotulados observados, 
buscando formas de consumo más saludables y adhiriendo a preparaciones con semillas, algunos cereales y legumbres sobre la base de lo aprendido.

En un espacio de intercambio constante la valoración de los objetivos medibles fue dándose en forma natural y como parte del proceso. Según el criterio de evaluación continua pudo en cada clase apreciarse el grado de avance en el conocimiento, las destrezas, la toma de conciencia y la tendencia al cambio de los comportamientos alimentarios en los alumnos, aunque es necesario reconocer que es dificultoso evaluar los cambios de hábitos alimentarios a largo plazo. Así, se llegó a una última clase práctica de evaluación integradora final.

La evaluación final consistió en la elaboración grupal de un menú, como método para evaluar los aprendizajes logrados en base a las estrategias aplicadas. Los objetivos primordiales a evaluar fueron la capacidad y autonomía de los participantes para tomar decisiones acerca de la elección de alimentos saludables, las combinaciones adecuadas de los ingredientes y la capacidad de manipulación higiénica de los alimentos. En función de lo aprendido durante las clases, los alumnos debían:

1. Proponer un menú que fuese práctico, fácil de realizar, saludable, apetitoso y adaptado al estilo de vida universitario, por lo cual además debía cumplir el requisito de ser fácil de trasladar y consumir entre horas de clases.

2. Investigar sobre las propiedades nutricionales y funcionales de los ingredientes utilizados, el contenido en micro y macronutrientes, las porciones recomendadas y las formas de conservación para prolongar la vida útil del menú elaborado.

3. Llevarlo a cabo en el tiempo de la clase (3 horas).

La totalidad de los participantes pudo resolver el desafío en forma exitosa.

No menos importante fue la evaluación, por parte de los estudiantes, hacia el curso formativo en todos sus aspectos. Para ello se recurrió a encuestas de tipo «múltiple opción» y de preguntas abiertas, que permitiesen la continuidad y/o mejoramiento de las estrategias implementadas. Según lo encuestado:

El material resultó atractivo, muy comprensible y muy motivador para realizar modificaciones en los hábitos alimentarios, sugiriéndose subir más recetas. En relación a la capacidad para promover una mejor alimentación, respetando las costumbres y cultura, se lo calificó como adecuado y muy adecuado. Mencionaron que les permitió conocer cómo organizar un menú nutritivo y sano, diario o semanal de rápida preparación y económico. El vocabulario y expresiones utilizadas fueron calificados como muy adecuados. Entre los aspectos que manifestaron como aportes novedosos a su conocimiento se mencionaron: conocimiento de la existencia de nuevos alimentos y sus 
propiedades (nutricionales, químicas y biológicas); concientización sobre las indicaciones de las GAPA. Respecto de las prácticas: manejo de los alimentos para evitar pérdida de nutrientes y la importancia del conocimiento de las diferentes formas de cocción; la incorporación en un mismo plato de varios ingredientes, su procesamiento y tipo de preparación; la incorporación de frutas y verduras a la dieta en formas diferentes y novedosas. Algunas opiniones compartidas en comunicaciones sociales se muestran en la Figura 2.
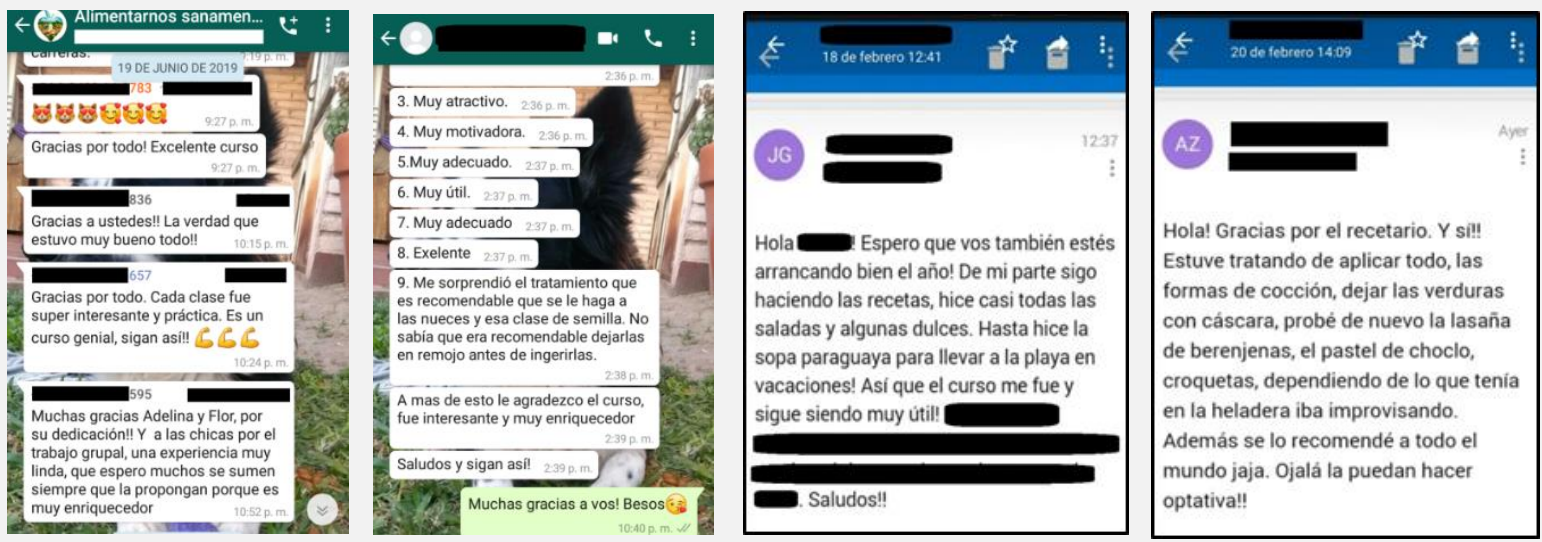

Figura 2. Opiniones compartidas con los alumnos sobre su experiencia en el curso.

\section{La mirada desde una formación extracurricular en docencia...}

Considerando la importancia que revistió para el desarrollo del curso la formación de nuevos recursos humanos capaces de promover desde lo educativo la alimentación saludable, a continuación, se desarrolla la narrativa de la experiencia (en 2019) relatada por la alumna de Licenciatura en Nutrición que formó parte del equipo docente.

Desde la mirada del alumno-docente se valoró la formación extracurricular en docencia como posibilidad de pensar la práctica y de pensarse a sí mismo ejerciendo un rol docente, y como herramienta útil para la construcción de experiencias propias que sirvan a una preparación futura. Baraldi et al. (2012:140) realzan la importancia de las experiencias previas en docencia como predisponentes de las experiencias futuras, apoyándose en las reflexiones de Dewey, quien afirma: «El educador dirige su mirada hacia adelante y contempla toda experiencia presente como una fuerza activa que influye en lo que serán las experiencias futuras».

Un primer objetivo fue la exploración y aproximación a la función docente, entendiéndola como quien se debe formar para luego asumir la responsabilidad de formar al otro, aprovechando el escenario creado en el aula como un espacio para el desarrollo de competencias necesarias para un futuro desempeño docente eficaz y un crecimiento mutuo (docente-alumno). Paralelamente, la 
adquisición de habilidades de comunicación para la construcción de conocimientos y el diseño de material didáctico que despierte la atención de la población destinataria, son de fundamental importancia en la formación de los profesionales de la salud que se desempeñen tanto en el ámbito clínico como en el académico.

Según Holli y Beto:

Los estudios muestran que mientras más activo sea el involucramiento de la persona en el proceso de aprendizaje, mejor será la retención (...) leer la información no es tan productivo como verla y escucharla o, mejor aún, discutirla o hacer algo con ella. (2018:304)

Este concepto se utilizó como pilar para el abordaje de las clases y los aportes personales. Para cada propuesta innovadora planteada se tuvo en cuenta el propósito del curso, los objetivos, las preferencias del estudiante, las instalaciones disponibles, el tiempo, costo de los ingredientes para los menús, etc., eligiendo siempre aquellas prácticas que requiriesen la participación activa del alumno para asegurar no solo un interés constante, sino también un cambio actitudinal en su estilo de vida. De este modo, la contribución se vio reflejada en las diferentes etapas: diagnóstica (registro de ingesta de 24 horas y cuestionario de frecuencia alimentaria), el proceso educativo y su proyección en los hábitos de alimentación saludable (nuevo recetario con rótulos) y la evaluación del curso. Para saber si las estrategias empleadas resultaron eficaces o si es necesario hacer modificaciones se trabajó con el propósito de valorar la calidad de las estrategias implementadas y el grado de acercamiento a los objetivos propuestos; entendiendo la evaluación como un sistema de retroalimentación en la relación evaluador-estudiante.

\section{Conclusiones}

La alimentación inadecuada incide en patologías de crecimiento exponencial en la actualidad; es por ello que resulta necesaria la implementación de estrategias educativas centradas en la modificación del estilo de vida hacia hábitos más saludables, particularmente cambios en las conductas alimentarias.

El ámbito académico cumple un papel de relevancia para promover la educación alimentaria nutricional y la salud integral de su comunidad. De este modo, el curso Alimentarnos Sanamente representa una estrategia valiosa para alumnos universitarios ya que brinda conocimientos teóricos basados en las GAPA, acompañados de una importante práctica contextualizada. La limitación en este punto, es que los resultados obtenidos dan cuenta de los logros en el corto plazo, sin poder establecer si estos conocimientos y aptitudes serán 
mantenidos en el tiempo. Puede concluirse que se considera beneficioso replicar estas estrategias educativas y, más aún, hacerlas extensivas a otros actores interesados de la comunidad universitaria.

\section{Agradecimientos}

Esta investigación se llevó a cabo en el marco del Programa CAl+D (Curso de Acción para la Investigación y Desarrollo) financiado por la Universidad Nacional del Litoral; Proyecto CAI+D 2016-UNL PI 50120150100042LI. 


\section{Referencias bibliográficas}

- Administración Nacional de Medicamentos, Alimentos y Tecnología Médica. Nuevo rotulado nutricional. Código Alimentario Argentino.

http://www.anmat.gov.ar/consumidores/Rotulado_nutricional.pdf

- Baraldi, V.; Bernik, J.; Díaz N. (2012). Una didáctica para la formación docente: dimensiones y principios para la enseñanza. Ediciones UNL.

- Berta, E.; Ávila, O.; Cúneo, F.; Vargas, M. y Fortino, M.A. (2017). Prácticas alimentarias y factores de influencia en estudiantes del segundo año de las carreras de la Facultad de Bioquímica y Ciencias Biológicas. FABICIB, (21), 130. Sección Resúmenes de Congreso. XL Reunión de CASLAN y VII Congreso de Alimentos del siglo XXI: Alimentos, Nutrición y Salud.

- Bordas, M.; Cabrera, F. (2001). Estrategias de evaluación de los aprendizajes centrados en el proceso. Revista española de pedagogía, (228), 25-48.

- Cardozo-Ortiz, C. (2011). Tutoría entre pares como una estrategia pedagógica universitaria. Educación y Educadores, 14(2), 309-325.

- De Girolami, D. y González Infantino, C. (2008). Clínica y Terapéutica en la Nutrición del Adulto. El Ateneo.

- Díaz Barriga Arceo, F.; Hernández Rojas, G. (2010). Estrategias para un aprendizaje significativo. Una interpretación constructivista. Mc Graw Hill.

- Fernández García, I. (2003). La educación entre pares: Los modelos del alumno ayudante y mediador escolar. Milenio revista digital.

http://www.deciencias.net/convivir/1.documentacion/D.ayuda.iguales/Educacion_entre_pare s(Fernandez)6p.pdf

- Flores, J.; Caballero Sahelices, M.C.; Moreira, M.A. (2009). El laboratorio en la enseñanza de las ciencias: Una visión integral en este complejo ambiente de aprendizaje. Revista de Investigación, 68(33), 75-112.

- Fortino, M.; Vargas, M.; Gavilán, L.; Celeghin, A.; Ortigoza, L. y Ávila, O. (2019). Educación en alimentación saludable: factores condicionantes en una experiencia de docenciainvestigación. Aula Universitaria, (20). https://doi.org/10.14409/au.v0i20.7718

- Holli, B. y Beto, J. (2018). Educación nutricional. Guía para profesionales de la nutrición. Wolterskluwer.

- Ibáñez Bernal, C. (2007). Un análisis crítico del modelo del triángulo pedagógico. Una propuesta alternativa. Revista Mexicana de Investigación Educativa., 12(32), 435-456. México.

- Ministerio de Salud de la Nación (2016). Guías Alimentarias para la Población Argentina. Buenos Aires. 
- Ministerio de Salud y Desarrollo Social (2019). 4ta. Encuesta Nacional de Factores de riesgo. Principales resultados.

http://www.msal.gob.ar/images/stories/bes/graficos/0000001444cnt-2019-04_4ta-encuestanacional-factores-riesgo.pdf

- Noseda, J.; Cúneo, F.; Fortino, M.A. (2019). Valoración del interés y conocimiento acerca de alimentación y actividad física en estudiantes del ciclo básico de la Facultad de Bioquímica y Ciencias Biológicas-UNL. FABICIB, (22), 1-15. Doi: 10.14409/fabicib.v22i1

- OMS (Organización Mundial de la Salud) (2017). 10 datos sobre obesidad. https://www.who.int/features/factfiles/obesity/es/

- OMS (Organización Mundial de la Salud) (2018). Obesidad y Sobrepeso. https://www.who.int/es/news-room/fact-sheets/detail/obesity-and-overweight

- Vargas, M.; Noseda, J.; Cúneo, F.; Berta, E.; Ávila, O.; Gavilán, L.; Fortino, M.A. (2017). Evaluación de la actividad física e ingesta de bebidas en estudiantes de la Facultad de Bioquímica y Ciencias Biológicas-UNL. FABICIB, (21), 130-131. 